\title{
Whole grain and technological aspects: example of biscuits and bread substitutes
}

\author{
Aliette VEREL, Catherine GERARD
}

Carbohydrates are the single most important source of food energy in the world. They comprise some 40 to 80 percent of total food energy intake, depending on local, cultural considerations or economic status. Carbohydrates have a wide range of physiological effects which may be important to health, such as provision of energy as mentioned above, and also effects on satiety/gastric emptying, control of blood glucose and insulin metabolism, cholesterol and triglyceride metabolism... (FAO/WHO, 1998).

Based on the world production, the major source of carbohydrates is cereals (almost $50 \%$ ) and consequently, cereal products are food of prime importance regarding the carbohydrate intake as they represent an important part of a daily meal.

A biscuit portfolio shows large variations of carbohydrate supply - from 20 to 90 percent depending on the biscuit type; The talk will be focused on bread substitutes and biscuits which are consumed breakfast and for afternoon snack. These products are the results of complex associations and interactions between proteins, lipids, carbohydrates (starch, soluble and insoluble fibres, ...) and water, occurring differently with process parameters (fermentation, mixing, baking, ....). They contain $20 \%$ to $70 \%$ cereals, for biscuits, and $75 \%$ to $100 \%$ cereals for bread substitutes.

In these products, cereals are mainly brought by wheat flours which are selected for their technological properties and which can be more or less extracted, more or less refined. Some specific biscuits or bread substitutes are real source of carbohydrates $(>50 \%)$, providing mainly from white wheat flour, in order to answer the consumer demand. The history of refined and whole grain breads and baked products reflects the belief that "white" flour was the food of the rich and unrefined flour was the food of the hard-working peasant and the poor. Consequently, for millennia, "white" flour was considered to be the pure flour of the rich and powerful and whole-grain flour was considered to be for peasants and there is no doubt that people wanted to get whiter and whiter flours. Moreover, white flours are excellent sources of carbohydrates, bring a part of the grain fibres and provide a low fat intake, despite missing a part of the nutritional interest of cereals.

Over the coarse of history, the topic of whole grains and health has evolved and in the early to mid-1990s, the importance of whole grain and health was once again rekindled to explore the relationship between whole grains, heart disease, and cancer. Considerable research suggests that consumption of whole grains reduces risk for heart disease, some cancer, and type-2 diabetes? The mechanisms responsible for the health benefits of whole grains are not clear. However, whole grains contain concentrated sources of dietary fibre, vitamins, trace minerals, phenolic compounds... Whole-grain constituents may work synergistically to mediate cholesterol, insulin and glucose responses; decrease blood pressure; function as antioxydants; bind carcinogens; and decrease transit time through the colon. The emerging science of whole grains suggests that it is necessary to eat the entire "fibre complex" (bran, germ, and endosperm), with all its biologically active compounds, not just the "fibre" portion of the 
plant food. There is little doubt today that whole grains and whole-grain products are far superior to white flour or other refined grain products in disease prevention and good nutrition, but it is difficult to overcome the age-old concept, deeply ingrained in the minds of many people, that white flour is the best possible choice. The challenge for the food industry is to bring back whole-grain flour, whole grains and whole-grain products as the first choice.

As a consequence, to valorise the nutritional quality of whole-wheat and actively propose solutions in the field of public health, we want to introduce macro and micro nutrients of wheat to maximise nutritional properties of our products while keeping good sensory quality for the consumer. However, we face a difficulty as traditional whole wheat flours are not adapted to biscuits and bread substitutes processing because they alter dough forming, products leavening, texture and sensory properties (Fig.1).
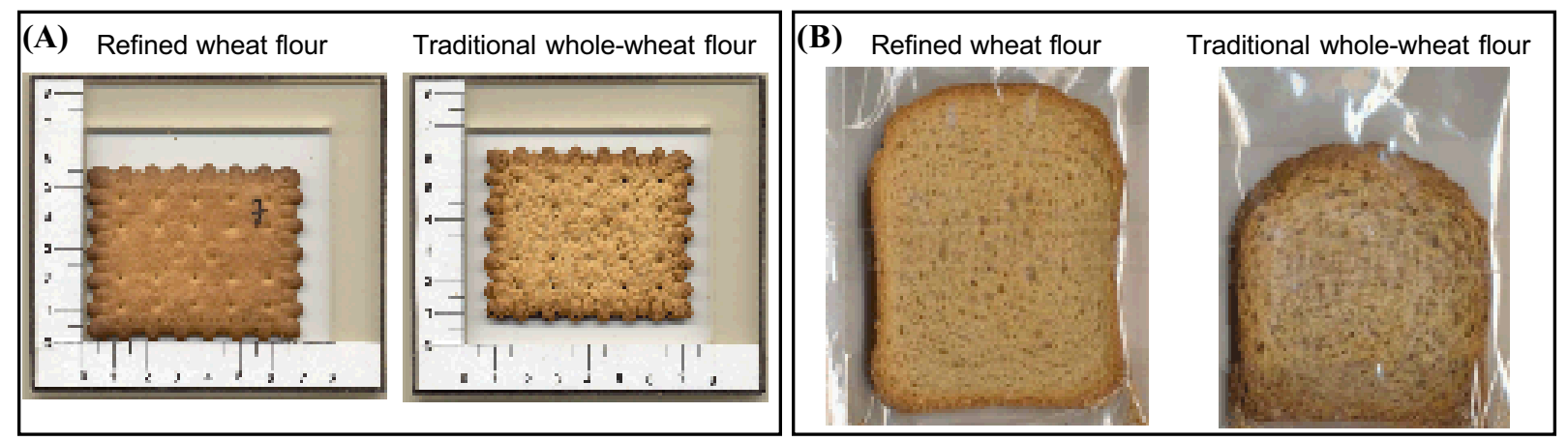

Figure 1 : photographs of biscuits (A) and bread substitutes (B) prototypes processed with refined wheat flour and traditional whole-wheat flour (35\% in the finished product).

To solve this problem, alternative cereal sources were explored on both whole-grain cereals, differing in type (flours, flakes...) and source (wheat, oat, rye, barley...), and specific cereal fractions such as middling, coarse bran, fine bran or others products issued from the milling process. Concomitantly, the nutritional interest of each of these alternatives was evaluated in terms of macro- and micro-nutrients content and relevant physico-chemical parameters were established regarding the technological behaviour. In the field of wheat, which is of prime importance for the biscuit area, methods of reconstitution of a whole-wheat flour were deeply assessed in order to get complete kernel, in respect with the histological parts of the kernels (endosperm, bran and germ) and the traditional milling process.

Obviously, technological behaviour of each alternative was measured using baking tests for rotary molded and sheeted biscuit models. The baking tests used were completely standardised (recipe - kneading, shaping, baking parameters) and allowed to produce $500 \mathrm{~g}$ of dough and 9 biscuits. The dough processability was established by cohesiveness and stickiness scores and the biscuit quality was measured via numerous criteria : density, thickness, length, width, weight, aspect and hardness.

This complete and multi-disciplinary approach allowed us to explain why combinations of refined wheat flours, whole wheat flours, brans, germs, ...with optimised rates adapted to biscuits and bread technologies are interesting. Examples will be presented to show how we can provide nutritional properties of whole grains in biscuits and bread substitutes while preserving the sensory quality and consumer preference. 


\section{Conclusion :}

Refined wheat flours have physico-chemical characteristics totally adapted to biscuit and bread substitutes processing, allowing to commercialise products with a high sensorial quality (aspect, texture and taste). However, the use of such flours prevent the valorisation of the whole nutritional benefits of cereals. In order to produce biscuits with a better nutritional composition (fibres, vitamins, minerals...), Danone Research has studied means to introduce whole grains without any degradation of the organoleptic quality of the products. The use of reconstituted whole-wheat flours allows to process cereal products showing an enhanced nutritional balance while keeping an acceptable organoleptic quality for consumers. The use of active fractions of cereals is promising for the processing of functional products which specific benefits will have to be demonstrated by clinical studies.

FAO/WHO. Carbohydrates in human nutrition: Report of a Joint FAO/WHO Expert Consultation. 14-18 April 1997, Reprinted 1998, Rome. FAO Food and Nutrition Paper No 66. (http://www.fao.org/docrep/w8079e/w8079e00.htm\#Contents). 